\title{
Michaela Tscherne
}

Pädagogische Hochschule Niederösterreich, Campus Baden

\section{Von Perspektive zu Perspektive}

\section{Eine Renaissance der Bildung wird eingeläutet}

DOI: https://doi.org/10.53349/sv.2021.i1.a73

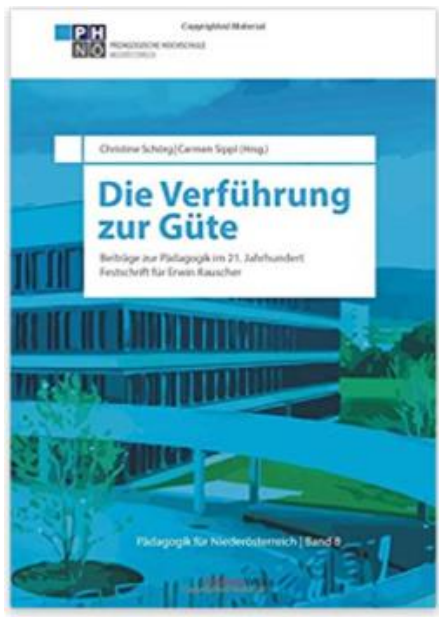

Christine Schörg \& Carmen Sippl (Hrsg.)

Die Verführung zur Güte

Beiträge zur Pädagogik im 21.

Jahrhundert

Festschrift für Erwin Rauscher

StudienVerlag, 2020

ISBN 978-3-7065-4967-7

Perspektive formt Raum.

Raum macht Lernen.

Lernen ändert Verhalten.

Verhalten prägt Gesellschaft.

Gesellschaft braucht Perspektive.

(Erwin Rauscher anlässlich der Eröffnung des Neubaus der Pädagogischen Hochschule Niederösterreich in Baden am 24. Mai 2019) 


\section{Impulse für die Pådagogik im 21. Jahrhundert}

Christine Schörg und Carmen Sippl versammeln in der von ihnen herausgegebenen Festschrift für Erwin Rauscher Beiträge, die Impulse für die Pädagogik geben sollen, um eine Renaissance der Bildung zu postulieren und gleichsam einzuläuten. Ilse Schrittesser, Konrad Paul Liessmann, Reinhold Leinfelder oder Michael Schratz, um hier nur einige der namhaften Autor*innen zu nennen, richten in ihren Beiträgen ihren Blick aus verschiedenen Perspektiven auf die Pädagogik der Zukunft und orientieren sich dabei an den von Erwin Rauscher formulierten Leitsätzen „Perspektive formt Raum - Raum macht Lernen - Lernen ändert Verhalten - Verhalten prägt Gesellschaft - Gesellschaft braucht Perspektive“. Diese bilden gleichsam die thematische Ausrichtung für den Aufbau des Buches. Nach einer Hinund Einführung der Herausgeberinnen in die Thematik folgen zunächst Glückwünsche von Johanna Mikl-Leitner, Christiane Teschl-Hofmeister, Christa Schnabl und Arthur Mettinger, bevor die Leitsätze von den Autor*innen aus ihrer Sicht diskutiert werden.

Der Titel „Die Verführung zur Güte“ offenbart die Absicht des Sammelbandes und soll gleichsam als Auftrag der Pädagogik gesehen werden, nämlich Mut zu machen für das Probieren und Erforschen, dafür, die Leidenschaft für das Neue zu erzeugen und zu teilen, aber vor allem darum, die besten Bedingungen für das Lernen zu schaffen. Bereits in den ersten Kapiteln wird klar, dass - ganz im Sinne von Erwin Rauscher - eine "Wiedergeburt" der Bildung im 21. Jahrhundert eingefordert wird, wobei den Leser*innen eine Vielfalt an Themenfeldern geboten wird, welche die Forschungstätigkeit des Jubilars im Laufe seines pädagogischen Wirkens begleitet haben und die sich in seinen zahlreichen Publikationen wiederfinden: Schulpädagogik, Schulentwicklung, Schulmanagement, Schulinnovation, Schulautonomie, Inklusion, Migration und Integration, Schulmathematik, Schulleitung und Führungskultur, Theater/Kunst/Kultur und Religionspädagogik.

Beispielhaft sei hier ein Beitrag von Michael Schratz vorgestellt, der sich dem Thema "Schule aus der entstehenden Zukunft entwickeln: Auf dem Weg in eine neue Führungskultur" widmet. Schratz plädiert für ein lernseitiges Führungsverständnis und identifiziert drei Schlüsselbereiche, welche die wirksame Bewältigung der komplexen gesellschaftlichen Herausforderungen aus pädagogischer Sicht ermöglichen sollen: Menschenbild und Werte, Emergenz und Resonanz sowie Schul- und Führungskultur, die er in weiterer Folge näher beschreibt. Er geht dabei u.a. auf die Bedeutung der Vision ein, die er mit einem „Leitstern, der einem immer wieder den Weg weist" (S. 136) vergleicht, verschriftlicht im Leitbild einer Schule, dessen Bedeutung im Kontext von Schulentwicklung, Zielbildern und Wertvorstellungen anschaulich skizziert wird.

„Bildung zielt auf die Unverfügbarkeit und Einmaligkeit der Person ab.“ (S. 139). Damit leitet Schratz seine Ausführungen zum Themenkomplex Emergenz und Resonanz ein und appelliert, Prozesse und Strukturen zu schaffen, die es ermöglichen, die unterschiedlichen Bedürfnisse der Lernenden im Sinne von Chancengleichheit zu bedienen. In Bezug auf Schul- und Führungskultur geht Schratz auf förderliche Bedingungen und Faktoren ein, um nachhaltige Auswirkungen auf das Lernen zu erreichen. Dabei beschreibt er anschaulich die drei Ebenen 
des Lernens: das Lernen der Schüler*innen, das Lernen der Lehrer*innen und das Lernen der Organisation, die es zu verbinden gilt, denn das System Schule könne nur lernen, „wenn alle Akteure darin lernen" (S. 142). Die Schulleitung habe in diesem Zusammenhang für eine wirksame, auf Resonanz aufbauende Beziehung zu sorgen, getragen und gestützt von Dialogkultur, kollegialer Zusammenarbeit und gemeinsamen Werten. Schratz resümiert in seinen abschließenden Ausführungen, dass eine lernseitige Perspektive verstärkte Schülerbeteiligung zur Förderung der pädagogisch bedeutsamen Beziehungsdimension im Schul- und Unterrichtsgeschehen als zentralen Qualitätsfaktor voraussetzt.

Lassen Sie sich von diesem und weiteren vielschichtigen Beiträgen in diesem Sammelband dazu verführen, erfolgreich eigene innovative Wege in der Pädagogik des 21. Jahrhunderts zu beschreiten - ganz im Sinne einer nachhaltigen Lehr- und Lernkultur.

Auf https://www.ph-noe.ac.at/de/ph-noe/wir-ueber-uns/rektorat/wir-gratulieren.html finden Sie weitere Stimmen zur Festschrift, unter https://www.phnoe.ac.at/de/forschung/forschung-und-entwicklung/schriftenreihe-paedagogik-fuerniederoesterreich.html Informationen zur Schriftenreihe „Pädagogik für Niederösterreich“.

\section{Autorin}

\section{Michaela Tscherne, Prof. Dr. BEd MBA MSc.}

Professorin und Qualitätsbeauftragte an der Pädagogischen Hochschule Niederösterreich; Arbeits- und Forschungsschwerpunkte: Leadership, Schulautonomie, Personalentwicklung, Berufsorientierung; Publikationen im Bereich Leadership. Kontakt: michaela.tscherne@ph-noe.ac.at 